Gaussdome.bas is the listing of a Basic program which calculates the effective surface tension of a liquid over the range of wave vectors where the discrete structure of a liquid surface starts to be important. Thus it successfully models the experimental results obtained by Mora et al (Mora, S.; Daillant, J.; Mecke, K.; Luzet, D.; Braslau, A.; Alba, M.; Struth, B. Phys. Rev. Lett. 2003, $90,216101 / 1)$. The program was written using BBEdit, which has the advantage that the Tools menu can be set up to enable a listed program to be run on a Macintosh computer under chipmunk Basic, Chipmunk Basic is available free at: www.nicholson.com/rhn/basic/

10 window $1,1,90,62$ : cls

20

30 dim probe3 (100), probe6(100) 'energy probabilities

$40 \operatorname{dim} a(6,6), b(6), c(6), a a(6,6), b b(6), d 3(6), d 6(6)$

'coefficients in polynomial fit routine

$50 \mathrm{dim} e 3(10,100)$, e6 $(10,100), f 3(10,100), f 6(10,100)$ 'energies and force constants vs layers and height 60 dim height(100), f(100) dome and distribution of peak height $70 \mathrm{dim} h(100), r(100)$

Gaussian curve 80 dim z(100), deltaz(100) displacements vs radius $90 \operatorname{dim} \mathrm{x}(100), \mathrm{y}(100), \operatorname{mr}(14)$ 'peak height of Gaussian variables and nradius values $100 \mathrm{dim} c 1(5), \mathrm{c} 2(5), \mathrm{c} 3(5)$ 110 dim gratio3(100), gratio6(100) 120 dim kvalue(20), $\log 10 \mathrm{k}(20), \mathrm{k} \$(20)$ ' ordinary ' local 130 dim gamfac3(100), gamfac6(100), gamma3(100), gamma6(100)

$140 \mathrm{c} 1(0)=0: \mathrm{c} 2(0)=0: \mathrm{c} 3(0)=100$

$150 \mathrm{c} 1(1)=0: \mathrm{c} 2(1)=100: \mathrm{c} 3(1)=0$

$160 \mathrm{c} 1(2)=100: c 2(2)=0: c 3(2)=0$

$170 \mathrm{c} 1(3)=50: c 2(3)=0: c 3(3)=50$

$180 \mathrm{c} 1(4)=0: c 2(4)=50: c 3(4)=50$

$190 \mathrm{c} 1(5)=80: c 2(5)=50: c 3(5)=0$

$200 \operatorname{mr}(1)=100: \operatorname{mr}(2)=50: \operatorname{mr}(3)=25: \operatorname{mr}(4)=16: \operatorname{mr}(5)=12$ : $\mathrm{mr}(6)=10$ : 'dome radius values

$210 \operatorname{mr}(7)=9: \operatorname{mr}(8)=8: \operatorname{mr}(9)=7: \operatorname{mr}(10)=6: \operatorname{mr}(11)=5: \operatorname{mr}(12)=4$ : $m r(13)=3: \operatorname{mr}(14)=2$

220

$230 \mathrm{vf}$ \$ = "\#\#\#.\#\#" : ve\$ = "\#\#.\#\#E\#\#\#"

240

250 restart: 
$260 \mathrm{cls}$

270 print " Vertical displacement energy and force constants for a Gaussian dome"

280 print " Distances are measured in units of the molecular diameter sigma": print : print

290

300 twopi $=2 * \mathrm{Pi}: \mathrm{kbt}=8.314 \mathrm{e} 7 * 300 / 6.02205 \mathrm{e} 23$ : nradii $\$="$ nradius values:" : $\log f a c=1 / \log (10)$

310

320 gosub chooseliquid

330

340 nhsteps $\%=100$ : hrange=12 : nlayers $\%=3$ : $\operatorname{nrmax} \%=100$ :

ngamma $=0$ : gscale6=1 : gscale $3=1$ : nsave $=0$ :'values for the

first run

350

360 iplot=1 : jplot=0 : iread=0 : istandard=1 : iauto=0 :

iopen $1=0$ : iopen $2=0$ : iopen $3=0$ : ipmax $=0$ : iloop $=0370$

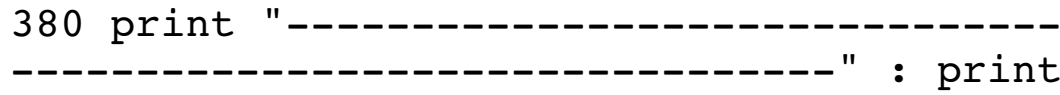

390 print "The first run uses a large dome radius $(>50)$ to obtain the scaling factor for gamma" : print

400 print " If the smallest e3 or e6 probabilities are greater than $1 \mathrm{e}-4$ "

410 print " the height range parameter probably needs

to be increased"

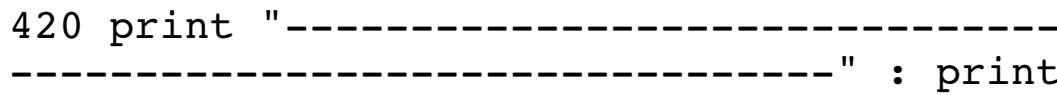

430

$440{ }^{\prime}========================$ get input parameters

$===================$

450

460 print "Print intermediate results (not recommended) $(\mathrm{y} / \mathrm{n})$ ?" : gosub keypress

465 if ucase $(y \$)=" Y "$ then ipmax $=1$

470 print : print "Loop over dome radius automatically

(recommended) ( $\mathrm{y} / \mathrm{n})$ ?"

475 gosub keypress : if ucase $(y \$)=" Y "$ then iloop=1

480 readlayers:

490 print : print "Current number of layers = "; nlayers\%

500 if iread=0 then print "OK? $(\mathrm{y} / \mathrm{n}) "$

510 gosub keypress : if ucase $(y \$)=" Y "$ then goto readheights

520 endif

530 input "Number of surface layers to use (range 2-10) ?" ; nlayers\%

540 if nlayers\% $>10$ or nlayers $\% 2$ then goto readlayers

550 if iread=1 then goto gaussian

560 readheights:

570 print "Current number of height steps = "; nhsteps\% 
580 if iread=0 then print "OK? $(\mathrm{y} / \mathrm{n})$ "

590 nst $\%=$ nhsteps $\% / 20+.5$

600 gosub keypress : if ucase\$(y\$)="Y" then goto readrange

610 endif

620 input "Number of height steps (range 10-100) ? "; nhs\%

630 if $\mathrm{nhs} \% 100$ or $\mathrm{nhs} \%<10$ then goto readheights

640 nhsteps $\%=$ nhs\%

650 nst $\%=$ nhsteps $\% / 20+.5$ : if iread=1 then goto gaussian

660 readrange:

670 print "Current maximum height in units of the rms height = "; hrange

680 if iread=0 then print "OK? $(\mathrm{y} / \mathrm{n}) "$

690 gosub keypress : if ucase $(y \$)=" Y "$ then goto readradius

700 endif

710 input "New maximum height (range 1 to 20 , not necessarily an integer)? "; hr

720 if $h r<1$ or $h r>20$ then goto readrange

730 hrange $=$ hr

740 if iread=1 then goto gaussian

750 readradius:

760 if iloop=1 then

770 print "Looping over fixed nradius values "

780 for $n l o o p=1$ to 14 : $n$ rmax $\%=m r(n l o o p)$ : goto gaussian

790 end if

800 print "Current dome radius in units of sigma $=$ "; nrmax\%

810 if iread $=0$ then

820 print "OK? (y/n)"

830 gosub keypress : if ucase $(y \$)=" Y "$ then goto gaussian

840 endif

850 input "New radius parameter (range 1-100) ? ";nr\%

860 if $n r \%<1$ or $n r \%>100$ then goto readradius

870 nrmax $\%=$ nr\% : goto gaussian

880

890 '===================== Loop over dome heights for fixed

nlayers\% $=================$

900

910 mainloop:

920 iread $=1$

930 npower $=5$

fifth-order polynomial fit

'use a

$940 \mathrm{nl}=\mathrm{nlayers} \%$

$950 \mathrm{kmin}=2.4048 /((\mathrm{nrmax} \%-.5) * \mathrm{sigma}): \mathrm{kmax}=4.8096 / \mathrm{sigma}$ :

kmecke $=\mathrm{Pi} /(($ nrmax $\%-.5) *$ sigma $)$

$960 \mathrm{kmax} \$=$ format $(\operatorname{kmax}, \operatorname{ve} \$)$ : $\operatorname{kmin} \$=$ format $\$(\mathrm{kmin}, \mathrm{ve} \$)$ : $\mathrm{kmecke} \$=$ format ( kmecke, ve\$ )

970 kvalue (ngamma) $=$ kmecke :

$\log 10 \mathrm{k}(\mathrm{ngamma})=\log (\mathrm{kvalue}(\mathrm{ngamma})) * \log f a c$

980 if iauto $=0$ then 
990 print : print "kmax, kmin, kmecke, kvalue:

"; kmax\$, kmin\$, kmecke\$, format\$ (kvalue (ngamma), ve\$ )

1000 print "ngamma = ";ngamma : print

1010 endif

1020 for $\mathrm{nh}=0$ to nhsteps\% : height(nh)=nh*hstep : 'use only positive heights, negative ones have same energy

1030 'print "step "; nh,"height = "; height(nh)

1040 for $n=0$ to $\operatorname{nrmax} \%: z(n)=h e i g h t(n h) * h(n): y(n)=z(n)$ :

$\mathrm{x}(\mathrm{n})=\mathrm{r}(\mathrm{n})$ : next $\mathrm{n}$ : 'height of element

1050 'if $n h>0$ then gosub drawplot

1060 'print "press any key" : gosub keypress

1070 for $n=0$ to $n \max \%-1$ : deltaz $(n)=z(n)-z(n+1)$ : next $n$

: 'height differences

1080

1090 power=3 : eflag=0

1100 e3 $(\mathrm{nl}, \mathrm{nh})=0$

1110 'print "nr, nmols\%, $\mathrm{PE}$ nmols\%": iswitch=1

1120 for $n r=0$ to $n r m a x \circ-1$ : d=deltaz $(n r)$

1130 if $n r=0$ then gosub PEcolumn : e3(nl, nh)=e3(nl, nh) +PE : nmols $\%=1$

1140 if $n r>0$ then gosub PEouter : nmols\%=twopi* $(\mathrm{nr}+1)$ : e3 $(n l, n h)=e 3(n l, n h)+P E * n m o l s \%$

1150 'print $\mathrm{nr}$; nmols\%; PE*nmols\%, : iswitch=-iswitch : if

iswitch=1 then print

1160 next $\mathrm{nr}$

1170 'print : print "e3 = ";e3(nl, nh)

1180

1190 power $=6$

1200 e6 $(\mathrm{nl}, \mathrm{nh})=0$

1210 'print "nr, nmolso, $\mathrm{PE} *$ nmols\%" : iswitch=1

1220 for $n r=0$ to $n r m a x \circ-1$ : d=deltaz(nr)

1230 if $n r=0$ then gosub PEcolumn : e6 $(\mathrm{nl}, \mathrm{nh})=\mathrm{e} 6(\mathrm{nl}, \mathrm{nh})+\mathrm{PE}$ :

nmols $\%=1$

1240 if nr>0 then gosub PEouter : nmols\%=twopi*nr :

e6 $(\mathrm{nl}, \mathrm{nh})=\mathrm{e} 6(\mathrm{nl}, \mathrm{nh})+\mathrm{PE} * \mathrm{nmols} \%$

1250 'print $\mathrm{nr}$;nmols\%; $\mathrm{PE} *$ nmols\%, : iswitch=-iswitch : if

iswitch=1 then print

1260 next $\mathrm{nr}$

1270 'print : print "e6, $\mathrm{nl}, \mathrm{nh}=$ ";e6(nl, nh);nl;nh

1280 next $\mathrm{nh}$

1290 if iauto=0 then print

"nh"; tab(12); "height"; tab(24); "e3" ; tab(36); "e6" ; tab( 48 ); "f3 " ; tab $(60) ; " \mathrm{f} 6 "$ "

1300 base $3=e 3(n 1,0)$ : base $6=e 6(n l, 0)$ : for $n h=0$ to nhsteps\%

1310 e3 $(\mathrm{nl}, \mathrm{nh})=$ base3-e3 $(\mathrm{nl}, \mathrm{nh}):$ e6 $(\mathrm{nl}, \mathrm{nh})=\mathrm{base} 6-\mathrm{e} 6(\mathrm{nl}, \mathrm{nh})$

1320 if e3(nl, nh) $<0$ or e6 $(\mathrm{nl}, \mathrm{nh})<0$ then eflag=1

1330 next $\mathrm{nh}$

1340 
1350 for $\mathrm{nh}=1$ to nhsteps\%

$1360 \mathrm{f} 3(\mathrm{nl}, \mathrm{nh})=2$ *e3 $(\mathrm{nl}, \mathrm{nh}) /$ height $(\mathrm{nh})^{\wedge} 2$

$1370 \mathrm{f} 6(\mathrm{nl}, \mathrm{nh})=2 * \mathrm{e} 6(\mathrm{nl}, \mathrm{nh}) /$ height $(\mathrm{nh})^{\wedge} 2$ : next $\mathrm{nh}$

1380 for $\mathrm{nh}=0$ to nhsteps\% step nst\%

1390 if iauto $=0$ then

1394 print

$\mathrm{nh}$; tab (12); height (nh); tab(24);e3(nl, nh); tab(36);e6 (nl, nh); tab (48

)$; f 3(\mathrm{nl}, \mathrm{nh}) ; \mathrm{tab}(60) ; \mathrm{f} 6(\mathrm{nl}, \mathrm{nh})$

1398 end if

1400 next $\mathrm{nh}$

1410 if eflag=1 and iauto=0 then

1420 print $" * * *$ Energy becomes negative $* * * "$ : print : iauto $=1$

1430 print "Adjusting maximum value of dome height" :goto

setheight

1440 end if

1450 if iauto=1 then goto setheight

1460 ncount $=0$

1470 gosub plots : if iloop=0 then print "Continue with these values $(y / n)$ ?"

1475 gosub keypress : if ucase\$(y\$)="N" then goto options

1480 gosub doaverage

1490 if ipmax=1 and iloop=0 then print "press any key"

1500 gosub getgamma

1510 if iloop $=1$ then next nloop : goto options

1520 print "Another nradius value $(y / n) "$ :gosub keypress

1530 if ucase $(y \$)=" N "$ then goto options

1540 goto readradius

1550

1560 ' $^{\prime}=============================$ adjust maximum height

automatically $==============$

1570

1580 setheight:

1590 ncount=ncount+1 : print ncount;

1600 if eflag=1 then

1610 hrange=hrange* .995

1620 if hrange $<1$ then hrange=1 : iauto=0 : print "At minimum value of height range"

1630 hstep=hrange*hrms/(sigma*nhsteps\%) : goto gaussian

1640 endif

1650 if $(\mathrm{f} 3(\mathrm{nl}, \mathrm{nhsteps} \%)<\mathrm{f} 6(\mathrm{nl}, \mathrm{nhsteps} \%)$ and $\mathrm{f} 3(\mathrm{nl}$, nhsteps\%) $>1)$

or $\mathrm{f} 6(\mathrm{nl}$, nhsteps\%) $<\mathrm{f} 3(\mathrm{nl}$, nhsteps\%) and $\mathrm{f} 6(\mathrm{nl}$, nhsteps\%) $>1$ then

1660 hrange=hrange $* 1.005$

1670 if hrange $>20$ then hrange=20 : iauto=0 : print "At maximum value of height range"

1680 hstep=hrange*hrms/(sigma*nhsteps\%) : goto gaussian

1690 endif

1700 iauto $=0$ : goto gaussian

1710 


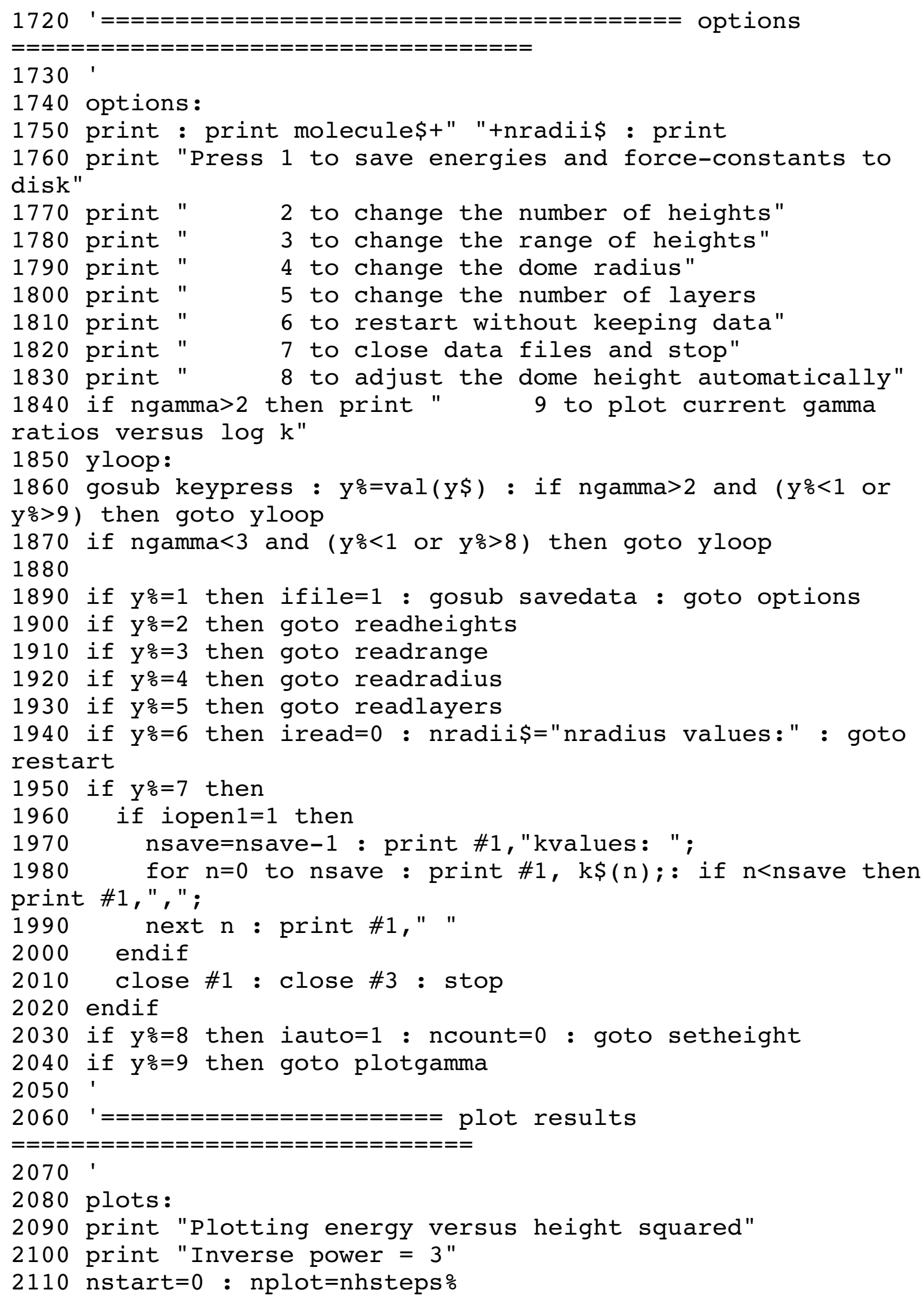


$2120 \mathrm{~b} \$="$ Potential versus height squared "+str\$(nl)+" layers, power $=3$ "

2130 for $n=n s t a r t$ to nhsteps $\% x(n)=\operatorname{height}(n)^{\wedge} 2: y(n)=e 3(n 1, n)$

: next $\mathrm{n}$

2140 iplot=1 : ncolor=0 : gosub drawplot : 'print "press any

key" : gosub keypress

2150 print "Inverse power $=6 "$

$2160 \mathrm{~b} \$=" P o t e n t i a l$ versus height squared "+str\$(nl)+" layers, power $=6 "$

2170 for $n=n s t a r t$ to nhsteps\% : $x(n)=$ height $(n)^{\wedge} 2$ : $y(n)=e 6(n 1, n)$

: next $\mathrm{n}$

2180 iplot=2 : ncolor=2 : gosub drawplot : if iloop=0 then print "press any key" : gosub keypress

2190 print "Plotting force constants versus height"

2200 print "Number of layers ";nl

2210 print "Inverse power $=3$ "

2220 nstart=1 : nplot=nhsteps\%

$2230 \mathrm{~b} \$="$ Force constant, number of layers $="+\operatorname{str} \$(n l)+"$,

inverse power $=3 "$

2240 for $n=n s t a r t$ to nhsteps\%: $x(n)=h e i g h t(n): y(n)=f 3(n l, n)$ : next $\mathrm{n}$

2250 iplot=1 : ncolor=0 : gosub drawplot : 'print "press any

key" : gosub keypress

2260 print "Inverse power $=6 "$

$2270 \mathrm{~b} \$="$ Force constant, number of layers $="+\operatorname{str} \$(\mathrm{nl})+"$,

inverse power $=6 "$

2280 for $n=$ nstart to nhsteps\% : $x(n)=$ height $(n): y(n)=f 6(n 1, n)$ :

next $\mathrm{n}$

2290 iplot=2 : ncolor=2 : gosub drawplot : return

2300

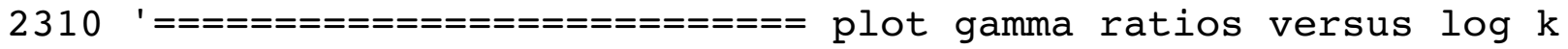

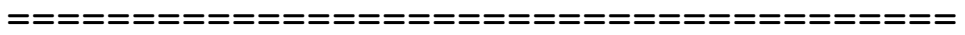

2320

2330 plotgamma:

2340 'plot program expects $x(n), y(n)$, nstart, nplot, iplot, jplot, and b\$

2350 print "Plotting gamma/gamma0 versus log k"

2360 print "Inverse power $=3$ "

2370 nstart=0 : nplot=ngamma-1

2380 for $n p=n s t a r t$ to nplot : $x(n p)=\log 10 k(n p)$ :

$\mathrm{y}(\mathrm{np})=\mathrm{gratio3}(\mathrm{np})$ : next $\mathrm{np}$

$2390 \mathrm{~b} \$="$ Gamma ratio versus $\log \mathrm{k}$, inverse power $=3$ "

2400 jplot=0 : iplot=1 : ncolor=0 : gosub drawplot

2410 jplot=1 : iplot=2 : gosub drawplot : print "press any key" : gosub keypress

2420 for $n p=n s t a r t$ to nplot : $x(n p)=\log 10 k(n p)$ :

$\mathrm{y}(\mathrm{np})=$ gratio6 $(\mathrm{np})$ : next $\mathrm{np}$

$2430 \mathrm{~b} \$="$ Gamma ratio versus $\log \mathrm{k}$, inverse power $=6 "$ 
2440 jplot=0 : iplot=1 : ncolor=2 : gosub drawplot

2450 jplot=1 : iplot=2 : gosub drawplot : iplot=1 : jplot=0 :

goto options

2460

2470 '==========potential energy calculation for the central

column, fixed $\mathrm{d}$, power and $\mathrm{nl}===========$

2480

2490 PEcolumn:

$2500 \mathrm{PE}=0$ : npo2=.5*power : for $\mathrm{n}=1$ to $\mathrm{nl}$ : rrsum $=0$

2510 rrsum=rrsum $+6 /\left(1+d^{\wedge} 2\right)^{\wedge}$ npo2 : 'attraction to one of $\mathrm{six}$ molecules at the same level initially

2520 rrsum=rrsum $+6 /\left(1+\left(1-d^{\wedge} 2\right)\right)^{\wedge}$ npo2 : 'attraction to one of $\mathrm{six}$ molecules in next lower level

2530 if $n>1$ then rrsum=rrsum+6/(1+(1+d^2) $)^{\wedge}$ npo2 : 'attraction to one of six molecules in next higher level

$2540 \mathrm{PE}=\mathrm{PE}+$ rrsum

2550 next $\mathrm{n}$

2560 return

2570 '

2580 '===== potential energy calculation for one molecule in an outer ring, fixed $\mathrm{d}$, power and $\mathrm{nl}======$

2590

2600 PEouter:

$2610 \mathrm{PE}=0$ : $\mathrm{npo} 2=.5$ * power : for $\mathrm{n}=1$ to $\mathrm{nl}$ : rrsum=0

2620 rrsum $=$ rrsum $+2 /\left(1+d^{\wedge} 2\right)^{\wedge}$ npo2 : 'attraction to two molecules at the same level initially

2630 rrsum=rrsum $+2 /\left(1+\left(1-d^{\wedge} 2\right)\right)^{\wedge}$ npo2 : 'attraction to two molecules in next lower level

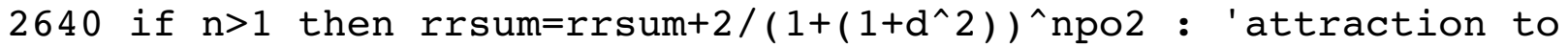
two molecules in next higher level

$2650 \mathrm{PE}=\mathrm{PE}+\mathrm{rrsum}$

2660 next $\mathrm{n}$

2670 return

2680

2690 ' ======================keypress routine

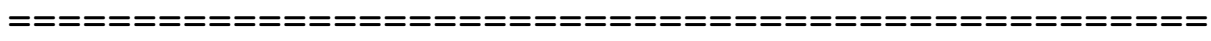

2700

2710 keypress:

$2720 \mathrm{y} \$="$ " : while y\$=" : $y \$=i n k e y \$$ : wend : return

2730 return

2740

2750 ' $======================$ Plotting routine

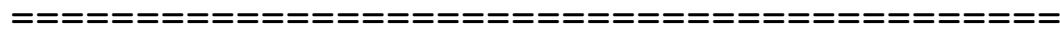

2760

2770 'expects $x(n), y(n)$, nstart, nplot, iplot, jplot, and b\$ 2780 drawplot:

2790 gosub sortx : 'sort data points into ascending order of $\mathrm{x}$ values 
2800 if iplot=2 then goto plotdata : 'don't redraw the axes or clear the rectangle

2805 hplot $=250:$ wplot $=300:$ graphics window $600,22,402,442$

: graphics color $90,90,90$

2810 graphics rect $20,20,400,440$ : graphics fillrect

$20,20,400,440,1$

2815 graphics fillrect $21,21,400,440,-1$ : graphics color $0,0,0$ 2820 graphics moveto 30,30 : graphics lineto 30,280 : graphics lineto 330,280

\section{0 plotdata:}

2840 graphics color c1(ncolor), c2 (ncolor),c3 (ncolor)

$2850 y \max =y($ nstart $): y \min =y \max : \operatorname{xmax}=x($ nstart $): \operatorname{xmin}=$ $\operatorname{xmax}: \operatorname{nmin}=$ nstart

2860 for $n=$ nstart+1 to nplot

2870 if $x(n)>x \max$ then $x \max =x(n)$

2880 if $y(n)>y \max$ then $y \max =y(n)$

2890 if $\mathrm{y}(\mathrm{n})<\mathrm{ymin}$ then $\mathrm{ymin}=\mathrm{y}(\mathrm{n}): \operatorname{nmin}=\mathrm{n}$

2900 if $x(n)<x$ min then $x$ min $=x(n)$

2910 next $n: y f a c=\operatorname{hplot} /(\operatorname{ymax}-y m i n): x f a c=\operatorname{wplot} /(x m a x-$

xmin)

2920 for $\mathrm{n}=$ nstart to nplot

$2930 \mathrm{nx}=30+\operatorname{int}(0.5+x f a c *(x(n)-x m i n))$

2940 ny $=280-i n t(0.5+(y(n)-y m i n) * y f a c)$

2950 if jplot=1 then graphics moveto nx, ny : graphics circle 6 :

goto skipline

2960 if $\mathrm{n}=\mathrm{nstart}$ then graphics moveto $\mathrm{nx}$, ny

2970 if $n>$ nstart then graphics lineto $n x, n y$

2980 skipline:

2990 next $\mathrm{n}$ : if jplot=1 then return

3000 graphics moveto 30,320 : if iplot $=2$ then graphics moveto 30,380

3010 graphics drawtext b\$+"

3020 a\$ = "Range of horizontal axis "+format $(x m i n, v f \$)+"$ to "+format\$ ( xmax, vf\$) +"

3030 graphics moveto 30,340 : if iplot $=2$ then graphics moveto 30,400

3040 graphics drawtext a\$

3050 a\$ = "Range of vertical axis "+format\$(ymin, ve\$)+" to

"+format\$ (ymax, ve\$) +"

3060 graphics moveto 30,360 : if iplot $=2$ then graphics moveto 30,420

3070 graphics drawtext a\$

3080 return

3090

3100 ' =========================== sort data points

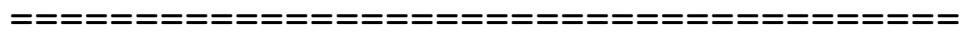

3110

3120 sortx: 
3130 for $n=$ nstart to nplot -1

$3140 \mathrm{xmin}=\mathrm{x}(\mathrm{n}): \mathrm{nmin}=\mathrm{n}$

3150 for $m=n+1$ to nplot : if $x(m)<x(n m i n)$ then $n$ min=m : iswap=1 : next m

3160 if iswap=1 then dum=x(n): $x(n)=x(\operatorname{nmin}): x(\operatorname{nmin})=d u m$ : $\operatorname{dum}=y(n): y(n)=y(n m i n): y(n m i n)=d u m \quad:$ iswap $=0$

3170 next $n$

3180 return

3190

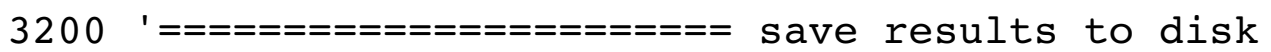

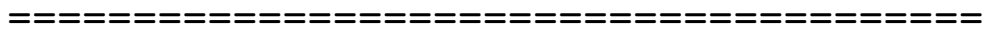

3210

3220 savedata:

3230 if ifile<>3 then

3240 if nsave=20 then print "k-value array is full" : return

3250 print : print molecules : print

3260 if iopen $1=0$ then

3270 input "Give a general title for the $1 / r^{\wedge} 3$ energy file "; title\$

3280 open "SFPutFile" for output as \#1 : print \#1,molecule\$+": "titles : iopen $1=1$

3290 endif

3300 for $\mathrm{nh}=0$ to nhsteps\% : print

$\# 1, \mathrm{nh} ; "$, " ; height (nh);"," ; height (nh)^2;

3310 for ncomma=0 to nsave: print\#1,","; : next ncomma

3320 print $\# 1, \mathrm{e} 3(\mathrm{nl}, \mathrm{nh})$; : for $\mathrm{n}=\mathrm{nsave}+1$ to 8 : print\#1,","; :

next $n$ : print\#1," "

3330 next nh : $\mathrm{k} \$($ nsave $)=$ kmecke\$ : print "nsave, $\mathrm{k}$ value:

";nsave;" ";k\$(nsave)

3340 if nsave $=20$ then print : print "*** Cannot save any more energy data***" : print : return

3350 nsave=nsave+1 : return

3360 endif

3370 if ifile=3 then

3380 if iopen $3=0$ then

3390 print molecules : print : input "Give a general title for the gamma ratio file ";titles

3400 open "SFPutFile" for output as \#3 : iopen3=1

3410 print \#3, molecule\$+": "+title\$

3420 print \#3,"Values of nlayers\%, nhsteps\%, hrange, hrms, sigma, gamma0, kmax, gscale3, gscale6:"

3430

print\#3, nlayers\%;" , " ; nhstepso;" , " ; hrange; " " ; hrms; " , ; sigma; " " ; gamma 0 ;", " ; kmax;" , " gscale3;" , " gscale6

3440 print\#3,"Values of ngamma, nrmax\%, kvalue, gamma3, gamma6, gratio3, gratio6:"

3450 endif

3460 print\#3, ngamma;"," ;nrmaxo;", " ;kvalue(ngamma);", " ; 
3465 print \#3,

gamma3 (ngamma); " , " ;gamma6 (ngamma) ; " , ";gratio3 (ngamma) ; ", " ;gratio 6 (ngamma)

3470 end if

3480 return

3490

3500 ' $======================$ average over distribution of

heights $=================================$

3510

3520 doaverage:

3530 gosub energyfit : print : p3sum=0 : p6sum=0 : p3min=1 : p6min $=1$

3540 e $3 \mathrm{rms}=0$ : for $\mathrm{m}=0$ to npower: $\mathrm{e} 3 \mathrm{rms}=\mathrm{e} 3 \mathrm{rms}+\mathrm{d} 3(\mathrm{~m}) * \mathrm{hrms} 2^{\wedge} \mathrm{m}$ :

next $\mathrm{m}: \mathrm{e} 3 \mathrm{fac}=.5 * \mathrm{kbt} / \mathrm{e} 3 \mathrm{rms}$

3550 e $6 \mathrm{rms}=0$ : for $\mathrm{m}=0$ to npower: $\mathrm{e} 6 \mathrm{rms}=\mathrm{e} 6 \mathrm{rms}+\mathrm{d} 6(\mathrm{~m}) * \mathrm{hrms} 2^{\wedge} \mathrm{m}$ :

next $\mathrm{m}: \mathrm{e} 6 \mathrm{fac}=.5 * \mathrm{kbt} / \mathrm{e} 6 \mathrm{rms}$

3560 for $n h=0$ to nhsteps\% : e3(nl, nh)=e3(nl, nh)*e3fac :

e6 $(\mathrm{nl}, \mathrm{nh})=\mathrm{e} 6(\mathrm{nl}, \mathrm{nh}) * \mathrm{e} 6 \mathrm{fac}$ : next $\mathrm{nh}$

3570 for $n h=0$ to nhsteps\% : $\operatorname{probe} 3(\mathrm{nh})=\exp (-\mathrm{e} 3(\mathrm{nl}, \mathrm{nh}) / \mathrm{kbt})$

3580 if probe $3(\mathrm{nh})<\mathrm{p} 3 \mathrm{~min}$ then $\mathrm{p} 3 \mathrm{~min}=\operatorname{probe} 3(\mathrm{nh})$

3590 if probe $3(\mathrm{nh})>\mathrm{p} 3 \mathrm{~min}$ then $\operatorname{probe} 3(\mathrm{nh})=0$

3600 p3sum $=$ p3sum + probe $3(\mathrm{nh})$

3610 probe $6(\mathrm{nh})=\exp (-\mathrm{e} 6(\mathrm{nl}, \mathrm{nh}) / \mathrm{kbt})$

3620 if probe $6(\mathrm{nh})<$ p6min then p6min=probe $6(\mathrm{nh})$

3630 if probe6 $(\mathrm{nh})>\mathrm{p} 6 \mathrm{~min}$ then $\operatorname{probe} 6(\mathrm{nh})=0$

3640 p6sum=p6sum+probe6(nh) : next $\mathrm{nh}$

3650 if ipmax=1 then

3660 print : print "Energies and un-normalised probabilities" : print

3670 print "height/sigma";tab(15);" e3";tab(30);" Prob e3"; tab(45);" e6";tab(60);" prob e6"

3680 for $n h=0$ to nhsteps\% step nst\%: print

format\$ (height (nh), ve\$); tab(15); format $(e 3(\mathrm{nl}, \mathrm{nh}), \operatorname{ve} \$)$; tab (30);

3690 print

format $(\operatorname{probe} 3(\mathrm{nh})$, ve\$); tab (45); format $(\mathrm{e} 6(\mathrm{nl}, \mathrm{nh})$, ve\$); tab (60);f ormat ( probe6 (nh), ve\$)

3700 next nh

3710 print : print "Energies and normalised probabilities" : print

3720 for $\mathrm{nh}=0$ to nhsteps\% : probe3(nh)=probe3(nh)/p3sum :

probe6 $(\mathrm{nh})=\operatorname{probe} 6(\mathrm{nh}) / \mathrm{p} 6 \mathrm{sum}:$ next $\mathrm{nh}$

$3730 \mathrm{p} 3 \mathrm{~min}=\mathrm{p} 3 \mathrm{~min} / \mathrm{p} 3 \mathrm{sum}: \mathrm{p} 6 \mathrm{~min}=\mathrm{p} 6 \mathrm{~min} / \mathrm{p} 6 \mathrm{sum}$

3740 print "height/sigma";tab(15);" e3";tab(30);" Prob e3"; tab(45);" e6"; tab(60);" prob e6"

3750 for $\mathrm{nh}=0$ to nhsteps\% step nst\%: print

format\$ (height (nh), ve\$); tab (15); format\$ (e3 (nl, nh), ve\$); tab (30); 
3760 print

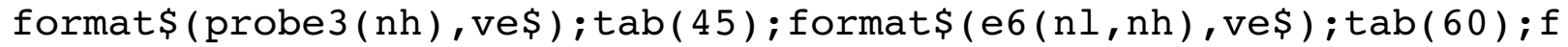
ormat $\$$ (probe6 ( $\mathrm{nh})$, ve\$ )

3770 next $\mathrm{nh}$ : print : return

3780 endif

3790 print "Initial, minimum and final probability values, inverse power $=3$ "

3800 print probe3(0), p3min, probe3 (nhsteps\%)

3810 print "Initial, minimum and final probability values, inverse power $=6 "$

3820 print probe6(0), p6min,probe6 (nhsteps\%)

3830 return

3840

3850 ' $=====================$ calculate apparent surface tension

at $\mathrm{kmin}============================$

3860

3870 getgamma:

3880 hsq3 $=0:$ hsq6 $=0$

3890 for $n h=0$ to nhsteps\%: hsq3=hsq3+probe3(nh)*height (nh)^2 :

hsq6=hsq $6+$ probe $6(\mathrm{nh}) *$ height $(\mathrm{nh})^{\wedge} 2:$ next $\mathrm{nh}$

$3900{ }^{\prime}$ gam3 $=\mathrm{kbt} /\left(\mathrm{pi} * \mathrm{hsq} 3 * \mathrm{sigma}{ }^{\wedge} 2\right): \operatorname{gam} 6=\mathrm{kbt} /\left(\mathrm{pi} * \mathrm{hsq} 6 * \mathrm{sigma}{ }^{\wedge} 2\right)$

3910 'print "Calculated gamma 3 and gamma6 values:

" ; format\$ ( gam3, "\#\#\#.\#"), format\$ (gam6, "\#\#.\#")

3920 gamfac3 (ngamma) $=$ hrms $2 /$ hsq 3 : gamfac $6($ ngamma $)=$ hrms $2 /$ hsq 6

3930 gamma3 (ngamma) = gscale $3 *$ gamma $0 *$ gamfac3 $($ ngamma $)$ :

gamma6 $($ ngamma $)=$ gscale6*gamma $0 *$ gamfac6 $($ ngamma $)$

3940 if istandard=0 then gratio3(ngamma)=gamma3 (ngamma)/gamma0 :

gratio6 ( ngamma) = gamma 6 ( ngamma ) / gamma 0

3950 if istandard=1 then

$3960 \quad$ gratio3 $($ ngamma $)=1$

3970

3980

gscale $3=$ gamma $0 /$ gamma 3 (ngamma)

3990 gratio6 $($ ngamma $)=1$

$4000 \quad$ gscale6=gamma $0 /$ gamma 6 (ngamma $)$

$4010 \quad$ gamma6 $($ ngamma $)=$ gamma 0

$4020 \quad$ istandard $=0$

4030 endif

4040 print : print "current ngamma = ";ngamma

4050 print

4060 print "kvalue, gamma3, gamma3/gamma0 =

"; kmin\$, gamma3 (ngamma), gratio3 (ngamma)

4070 print "kvalue, gamma6, gamma6/gamma0 =

"; kmin\$, gamma6 (ngamma), gratio6 (ngamma)

4080 if iloop=0 then print "Use these values $(y / n)$ ": gosub

keypress

4090 if ucase $(y \$)=" N "$ then return

4100 ifile=3 : gosub savedata : print "gamma value saved" 
4110 print : istandard=0 : nradii $\$=$ nradii\$+" "+str\$(nrmax\%) : ngamma $=$ ngamma +1 : return

4120

4130

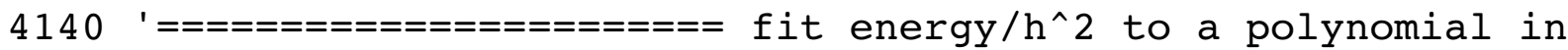

$\mathrm{h}^{\wedge} 2======================$

4150

4160 energyfit:

4170 print "Fitting energ/h^2 to a polynomial of order "; npower;"in h squared"

$4180 \mathrm{np}=$ nhsteps\% : nstart $=1:$ nplot $=\mathrm{np}: \mathrm{x}(0)=0: \mathrm{y}(0)=0$

4190 for $n=1$ to $n p: x(n)=h e i g h t(n)^{\wedge} 2$ : $y(n)=e 3(n l, n) / x(n)$ :

next $\mathrm{n}$

4200 gosub dosums : gosub gaussj : 'if ipmax=1 then gosub

checkfit : print "Results for inverse power $=3$ "

4210 for $n=1$ to npower : $d 3(n-1)=c(n)$ : next $n$

$4220 \mathrm{~b} \$=" F i t$ of e3/h-squared vs h-squared"

4230 jplot=0 : iplot=1 : ncolor=0 : gosub drawplot

4240 for $n=1$ to $n p: y(n)=0$ : for $m=1$ to npower+1 :

$\mathrm{y}(\mathrm{n})=\mathrm{y}(\mathrm{n})+\mathrm{c}(\mathrm{m}) * \mathrm{x}(\mathrm{n}) \wedge(\mathrm{m}-1)$ : next $\mathrm{m}$ : next $\mathrm{n}$

4250 jplot=1 : iplot=2 : gosub drawplot : if iloop=0 then print "press any key" : gosub keypress

4260 for $n=1$ to $n p$ : $y(n)=e 6(n l, n) / x(n)$ : next $n$

4270 gosub dosums : gosub gaussj : 'if ipmax=1 then gosub

checkfit : print "Results for inverse power $=6 "$

4280 for $n=1$ to npower : $d 6(n-1)=c(n)$ : next $n$

4290 b\$="Fit of e6/h-squared vs h-squared"

4300 jplot=0 : iplot=1 : ncolor=2 : gosub drawplot

4310 for $n=1$ to $n p: y(n)=0$ : for $m=1$ to npower+1 :

$\mathrm{y}(\mathrm{n})=\mathrm{y}(\mathrm{n})+\mathrm{c}(\mathrm{m}) * \mathrm{x}(\mathrm{n}) \wedge(\mathrm{m}-1)$ : next $\mathrm{m}$ : next $\mathrm{n}$

4320 jplot=1 : iplot=2 : gosub drawplot : iplot=1 : jplot=0 : if ipmax $=0$ then return

4330 print "Current order of the polynomial fit = "; npower : print

4340 print "Press another order for the polynomial (range 2 to 5 )

4350 print "or any value outside the range to continue " : if iloop $=0$ then gosub keypress

4360 npnew\%=val $(y \$)$

4370 if npnew\% $>1$ and npnew\%<6 then npower=npnewo : goto energyfit

4380 return

4390

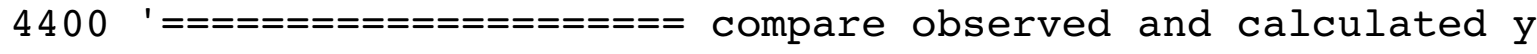

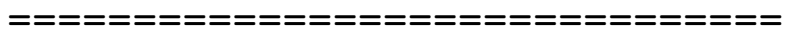

4410

4420 checkfit: 


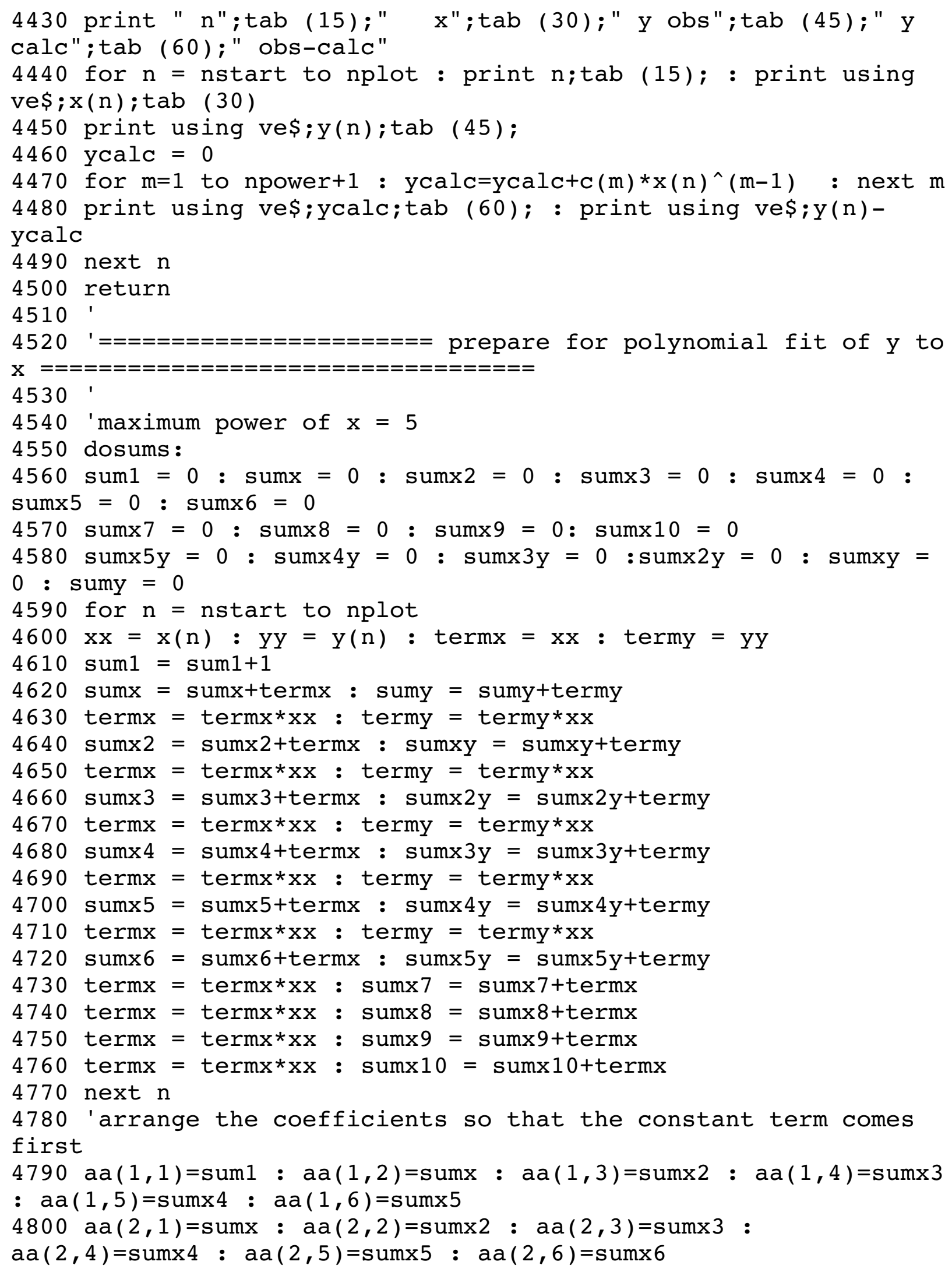


4810 aa $(3,1)=\operatorname{sumx} 2:$ aa $(3,2)=\operatorname{sumx} 3:$ a $(3,3)=\operatorname{sumx} 4$ : aa $(3,4)=\operatorname{sumx} 5:$ aa $(3,5)=\operatorname{sumx} 6:$ aa $(3,6)=\operatorname{sumx} 7$

4820 aa $(4,1)=\operatorname{sumx} 3:$ aa $(4,2)=\operatorname{sumx} 4$ : $a(4,3)=\operatorname{sumx} 5$ : aa $(4,4)=\operatorname{sumx} 6$ : aa $(4,5)=\operatorname{sumx} 7:$ aa $(4,6)=\operatorname{sumx} 8$

4830 aa $(5,1)=\operatorname{sumx} 4$ : aa $(5,2)=\operatorname{sumx} 5$ : aa $(5,3)=\operatorname{sumx} 6$ : aa $(5,4)=\operatorname{sumx} 7$ : aa $(5,5)=\operatorname{sumx} 8$ : aa $(5,6)=\operatorname{sumx} 9$

4840 aa $(6,1)=\operatorname{sumx} 5:$ aa $(6,2)=\operatorname{sumx} 6:$ aa $(6,3)=\operatorname{sumx} 7$ :

aa $(6,4)=\operatorname{sumx} 8: a a(6,5)=\operatorname{sumx} 9: a a(6,6)=\operatorname{sumx} 10$

$4850 \mathrm{bb}(6)=\operatorname{sumx} 5 \mathrm{y}: \mathrm{bb}(5)=\operatorname{sumx} 4 \mathrm{y}: \mathrm{bb}(4)=\operatorname{sumx} 3 \mathrm{y}: \mathrm{bb}(3)=$ sumx2y : bb(2) = sumxy : bb(1) = sumy

4860

4870 return

4880

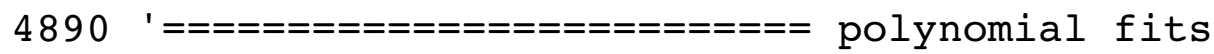

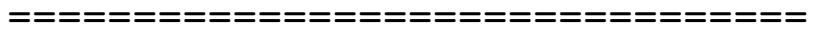

4900

4910 gauss $j:$

4920

$4930 \mathrm{~A}(\mathrm{n}, \mathrm{n})$ is the array of coefficients in the simultaneous equations

$4940 \mathrm{~b}(\mathrm{n})$ is the right-hand-side vector

4950 ' $\mathrm{c}(\mathrm{n})$ is the vector of solutions, $\mathrm{c}(1)$ being the constant

term

4960 'maximum power $=5$

4970

4980 for $n=1$ to $6: \mathrm{b}(\mathrm{n})=0$ : for $\mathrm{m}=1$ to 6 : $\mathrm{a}(\mathrm{n}, \mathrm{m})=0$ : next $\mathrm{m}$ :

next $\mathrm{n}$

4990 nmax=npower+1

5000 for $n=1$ to $n \max : b(n)=b b(n)$ : for $m=1$ to $n \max$ : $\mathrm{a}(\mathrm{n}, \mathrm{m})=\mathrm{aa}(\mathrm{n}, \mathrm{m})$ : next $\mathrm{m}$ : next $\mathrm{n}$

5010 if ipmax $=0$ then goto skipprint 1

5020 print : print "Initial matrix of coefficients:"

5030 for $\mathrm{n}=1$ to $\mathrm{nmax}$ : for $\mathrm{m}=1$ to $\mathrm{nmax}$ : print tab(10*(m-

$1))$; format $\$(a(n, m), v e \$)$; : next $m$

5040 print $\operatorname{tab}(10 * m+5)$; format $(b(n), v e \$)$ : next $n$

5050 print

5060 skipprint1:

5070 for $\mathrm{k}=1$ to $\mathrm{nmax}$ :

'row=k; elements before

$\mathrm{a}(\mathrm{k}, \mathrm{k})$ should be zero

$5080 \quad$ biga=abs $(a(k, k))$ : 'put the biggest element in

column $\mathrm{k}$ below row $\mathrm{k}$ into row $\mathrm{k}$

5090 if $\mathrm{k}<\mathrm{nmax}$ then

5100 for $\mathrm{n}=\mathrm{k}+1$ to $\mathrm{nmax}$

5110 if abs $(a(n, k))>b i g a$ then

$5120 \quad$ biga $=\operatorname{abs}(\mathrm{a}(\mathrm{n}, \mathrm{k}))$

5130 for $l=1$ to $\operatorname{nmax}: \operatorname{dum}=a(n, 1)$ :

$a(n, 1)=a(k, 1): a(k, 1)=$ dum : next 1 5140

$\operatorname{dum}=b(n): b(n)=b(k): b(k)=d u m$ 
5350 'now solve for unknowns and put them into the c array

5370 for $k=$ nmax to 1 step -1 : $c(k)=b(k)$ : 'print "row: "; $k$ : print $\mathrm{c}(\mathrm{k})$

5380 if $k<\operatorname{nmax}$ then

5390 for $m=k+1$ to $n \max : c(k)=c(k)-c(m) * a(k, m)$ : 'print $c(k)$, $\mathrm{c}(\mathrm{m}), \mathrm{a}(\mathrm{k}, \mathrm{m})$

5400 next $m$ : 'print

5410 endif

5420 next $\mathrm{k}$ : if ipmax=0 then return

5430 print "solutions:"

5440 for $m=1$ to $n \max$ : print tab(10*m); format $\$(c(m)$, ve $)$; : next $\mathrm{m}$ : print

5450 return 5460

5470 ' $=======================$ Calculate Gausian curve $============================$

5500 hstep=hrange*hrms/(sigma*nhsteps\%) : sigfac=3/nrmax\%^2 : $r(0)=0: h(0)=1$

5510 for $n=1$ to $n r m a x \%$ $5520 r(n)=n-.5: h(n)=\exp \left(-\operatorname{sigfac} * r(n)^{\wedge} 2\right)$ : next $n$ :'radius in units of sigma; go to 3 times rms width of Gaussian 
5530 for $n=0$ to $n \max \%: x(n)=r(n): y(n)=h(n):$ next $n$ : if iauto $=1$ then goto mainloop 5540 nstart $=0$ : nplot $=$ nrmaxo : $\mathrm{b} \$="$ Gaussian plot" : ncolor=3 : iplot=1 : jplot=0 : gosub drawplot

5550 'print "press any key" : gosub keypress

5560 goto mainloop

5570

5580 ' $==============================$ choose molecular

parameters $====================$

5590

5600 chooseliquid:

5610 print

5620 print "Press 1 for $\mathrm{H} 2 \mathrm{O} "$

5630 print " 2 OMCTS"

5640 print " 3 CCl4"

5650 print " 4 squalane"

5660 print " 5 glycol"

5670 print

5680 chloop:

5690 gosub keypress : iliq=val(y\$) : if iliq<1 or iliq>5 then goto chloop

5700 if iliq=1 then molecule $\$=" \mathrm{H} 2 \mathrm{O} "$ : sigma=2.5e-8 : gamma0=73 :

hrms $=1.34 \mathrm{e}-8:$ hrms $2=(\mathrm{hrms} / \mathrm{sigma}){ }^{\wedge} 2$

5710 if iliq=2 then molecule $\$="$ OMCTS":sigma=7.9e-

$8:$ gamma $0=18: \mathrm{hrms}=1.34 \mathrm{e}-8 * \operatorname{sqr}(73 / \mathrm{gamma} 0): \mathrm{hrms} 2=(\mathrm{hrms} / \mathrm{sigma}) \wedge 2$

5720 if iliq=3 then molecule $\$=" \operatorname{ccl} 4 ":$ sigma $=6.2 e-8:$ gamma $0=30$

: hrms $=1.34 \mathrm{e}-8 * \operatorname{sqr}(73 / \mathrm{gamma} 0): \mathrm{hrms} 2=(\mathrm{hrms} / \mathrm{sigma}) \wedge 2$

5730 if iliq=4 then molecule $\$="$ squalane" : sigma=1.01e-7 :

gamma $0=28: h r m s=1 \cdot 34 \mathrm{e}-8 * \operatorname{sqr}(73 /$ gamma 0$):$ hrms $2=(\mathrm{hrms} / \mathrm{sigma}) \wedge 2$

5740 if iliq=5 then molecule $="$ glycol":sigma $=4.4 e-$

$8:$ gamma $0=46:$ hrms $=1.34 e-8 * \operatorname{sqr}(73 /$ gamma 0$):$ hrms $2=(\text { hrms } / \text { sigma })^{\wedge} 2$

5750 return

5760 'First 3 from Mora et al; squalane from ellipsoid $1400 \mathrm{x}$

$950 \times 470 \mathrm{pm}$;

5770 'glycol from Hirschfelder,Curtiss\&Bird L-J data p1213,1214 